\title{
Le genre Bakalovaella BUCUR, 1993 (Dasycladeae, Dasycladaceae), et description de son plus ancien représentant crétacé
}

\author{
Bruno GRANI ER ${ }^{1}$ \\ I oan I. Bucur ${ }^{2}$
}

Résumé : Une nouvelle espèce de Dasycladacée de l'Hauterivien d'Aquitaine (France) est décrite. Elle est attribuée au genre Bakalovaella BUCUR, 1993, antérieurement mis à tort en synonymie avec le genre Montiella ( $L$. et J. MoReLLET, 1922). D'un point de vue phylogénétique, en se fondant sur le modèle du "piston élévateur", ce taxon appartiendrait à une lignée issue des Diploporacées.

\section{Mots-clefs :}

- algues vertes calcaires ;

- Dasycladales ;

- Cylindroporella ;

- Aquitaine ;

- Hauterivien :

- Barrémien

Citation: Granier B. \& Bucur I.I. (2019).- Le genre Bakalovaella Bucur, 1993 (Dasycladeae, Dasycladaceae), et description de son plus ancien représentant crétacé.- Carnets Geol., Madrid, vol. 19, no 1, p. 1-19.

Abstract: Reinstatement of the genus Bakalovaella BucuR, 1993 (Dasycladeae, Dasycladaceae), and a description of its earliest Cretaceous representative.- A new Dasycladacean species found in Hauterivian strata in Aquitaine is described. It is ascribed to the genus Bakalovaella BuCUR, 1993, which is reinstated here. From a phylogenetic point of view, based of the "lifting piston" model, this taxon should be part of a lineage derived from the Diploporaceae.

\section{Key-words:}

- calcareous green algae;

- Dasycladales;

- Cylindroporella;

- Aquitaine;

- Hauterivian;

- Barremian

\section{I ntroduction}

Dans le cadre d'une révision des algues cylindroporelliformes entreprise par le premier auteur (GRANIER, 1990, 2002, 2013, 2018; GRANIER et al., 1995, 2013, 2017) et de contributions sur le même sujet du second auteur (Bucur, 1993, 2011 ; Bucur et al., 2003), nous présentons tous deux ici une révision de sections erronément attribuées par Bouroullec et Deloffre (1968) à "Cylindroporella cf. sudgeni ELLIOTT, 1957".

1 Dépt. STU, Fac. Sci. Tech., UBO, 6 avenue Le Gorgeu, CS 93837, F-29238 Brest (France) bgranier@univ-brest.fr

2 Department of Geology and Center for Integrated Geological Studies, Babeş-Bolyai University, M. Kogălniceanu str., 1, 400084 Cluj-Napoca (Roumanie)

ioan.bucur@ubbcluj.ro

Manuscrit en ligne depuis le 17 janvier 2019

[Éditeur scientifique : Michel MOULLADE ; éditeur technique : Bruno GRANIER] 


\section{Matériel et méthode}

Pour des études micropaléontologiques, le matériel des Collections CuvilLieR, DeLOFFRE (S.N.P.A. - Société Nationale des Pétroles d'Aquitaine, devenue elf-Aquitaine) et J. EMBERGER constitue une ressource inestimable mais jusqu'à présent négligée. À l'occasion de prêts, il a été possible de consulter quelques lames minces du deuxième chercheur (R.D.) déposées au Centre Scientifique et Technique Jean FÉGER (TOTAL) à Pau (64, France). Quant aux lames minces des premier (J.C.) et troisième (J.E.) chercheurs, une partie est désormais déposée au "Laboratoire de $\mathrm{Pa}$ léontologie de Brest" (29, France). Ayant souvent été consultés comme experts par les compagnies pétrolières à des fins de datation biostratigraphique, les deux derniers chercheurs eurent ainsi l'occasion d'enrichir leurs collections personnelles de matériel provenant de forages et donc inaccessible au commun des géologues. Les lames minces étudiées proviennent de deux forages: Lacq 104 et Ousse 1, dont des fiches documentaires peuvent être trouvées sur le site web du B.R.G.M. - Bureau de Recherches Géologiques et Minières à l'aide de http://infoterre.brgm.fr/ search/search.htm

Les sections d'algues ont été systématiquement identifiées et photographiées au moyen d'un microscope stéréoscopique Olympus SZX7 avec un grossissement égal à $5.6 x$. Les mesures réalisées correspondent "classiquement" à $D$ (diamètre externe du manchon calcaire), $d$ (diamètre de l'axe principal), d* (diamètre de la cavité axiale), d/D et $d * / D$ en $\%$, e ou $L[=(D-d) / 2$, épaisseur du manchon calcaire ou longueur des latérales ], $I_{1}$ (Iongueur de la primaire stérile), $p_{1}$ (diamètre de la primaire stérile), $I_{2}$ (longueur de la secondaire stérile), $\mathrm{p}_{2}$ (diamètre de la secondaire stérile), $I_{f}$ (longueur de l'ampoule fertile), $p_{f}$ (diamètre de l'ampoule fertile), e (espacement des verticilles), $h$ (hauteur d'un verticille) et $e / h$ en $\%$ (imbrication des verticilles).

\section{Paléophycologie de 1968 à 2018 : 50 ans de questionnement}

En cinquante ans, nos connaissances sur les algues fossiles ont significativement progressé et il n'est guère étonnant que de nouvelles interprétations du matériel de Bouroullec et Deloffre (1968) aient déjà été proposées :
1. Macroporella embergeri BouRoulleC \& DeLofFRE, 1968 (op. cit., PI. 4), est devenue le type du genre Zergabriella GRANIER, 1989 ;

2. la plupart des sections attribuées à "Actinoporella podolica ALTH, 1878" (BOUROULLEC et Deloffre, 1968, PI. 5, figs. 1-3, 5-6, 9-10) correspondent pour l'essentiel à Pseudoactinoporella fragilis CONRAD, 1970 ;

3. les sections identifiées comme étant "Pianella dinarica (RADOIČIĆ, 1959)" (BOUROUlleC et DELOFFRE, 1968, PI. 7, figs. 1-8, 14-15), correspondent à diverses espèces dont des représentants du genre Salpingoporella (PIA in TRAUTH, 1918);

4. les sections attribuées à "Acicularia cf. elongata CAROzZI, 1947" (Bouroullec et DelofFre, 1968, PI. 7, figs. 9-13) correspondent à diverses espèces, dont des représentants du genre Terquemella MUNIER-CHALMAS ex L.et J. MORELLET, 1913.

En résumé, ce travail pionnier nécessitait une révision significative.

Le matériel analysé aujourd'hui ici provient des forages Lacq 104 (B.R.G.M., 2018a, La 104 :

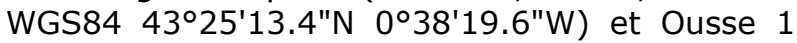
(B.R.G.M., 2018b, Ous 1: WGS84 43016'52"N $0^{\circ} 16^{\prime} 40^{\prime \prime} \mathrm{W}$ ) de la région de Pau, S Aquitaine (Fig. 1). Il correspond à des sections (BOUROULleC \& Deloffre, 1968, PI. 6, figs. 1-9) attribuées à "Cylindroporella cf. sudgeni ELLIOTT, 1957". Le terme "cf." invite à comparer ces sections à " $\mathrm{Cy}$ lindroporella sudgeni ELLIOT, 1957", une espèce récemment révisée par le premier auteur (GRANIER, 2018) et transférée à cette occasion dans le genre Holosporella PIA, 1930, car le thalle (pour sa partie fertile) est dépourvu de latérales stériles. Il n'y a pas de connexion directe entre l'axe principal et les ampoules fertiles contrairement à ce qu'envisageaient Bouroullec et DelofFre (1968, Fig. 3 ; ici : Fig. 2.A-B). Il ne s'agit donc pas non plus du genre Cylindroporella sensu JoHnson, 1954, dont les latérales stériles sont censées être disposées en alternance régulière avec des latérales fertiles au sein d'un même verticille ${ }^{[*]}$, mais de formes comparables à Montiella (L. et J. Morellet, 1922) ou mieux à Bakalovaella BUCUR, 1993 (Fig. 2.C-D). 


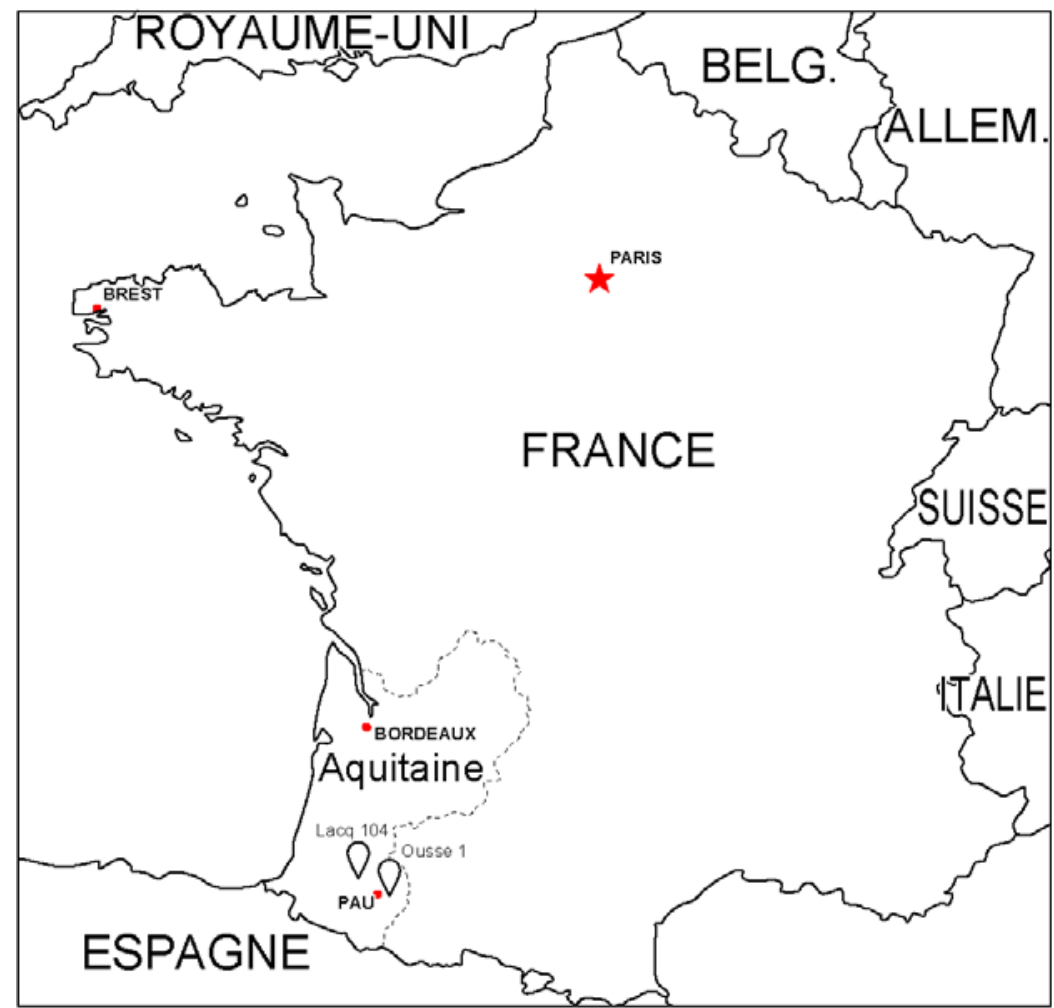

Figure 1 : Localisation des forages Lacq 104 (La 104) et Ousse 1 (Ous 1) en Aquitaine, SW France.
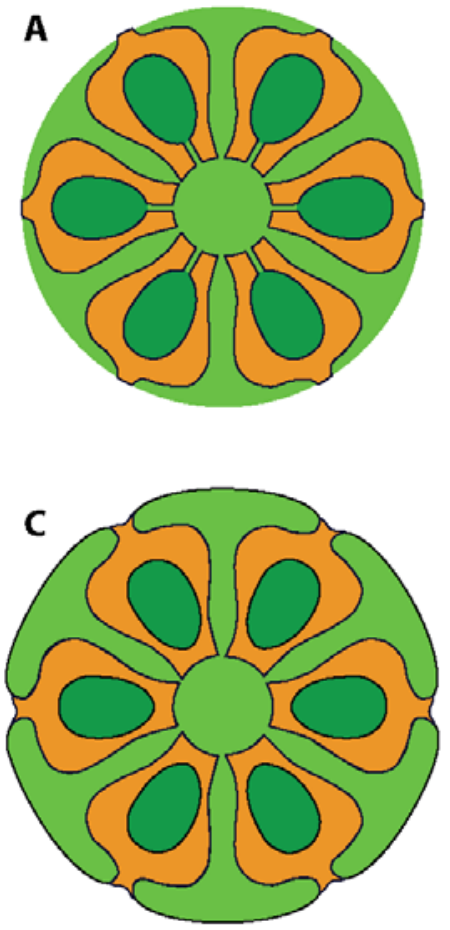

B

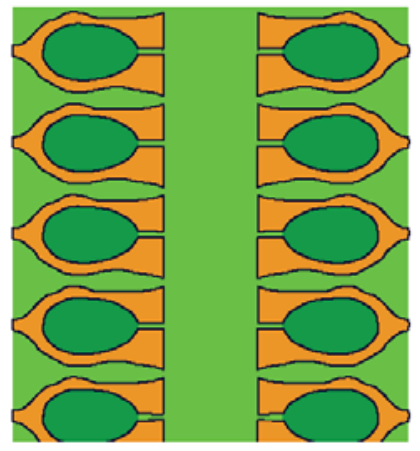

D

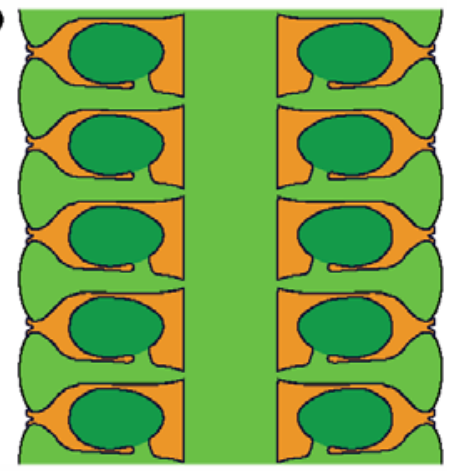

Figure 2 : A-B : reconstitution de "Cylindroporella cf. sudgeni ELLIOTT, 1957" d'après BoUROULLEC et Deloffre (1968, Fig. 3.1-2) ; C-D : reconstitution de Bakalovaella deloffrei n.sp. A, C : sections transverses ; B, D : sections axiales. En orange, la minéralisation; en vert clair, les parties stériles de l'algue; en vert foncé, les ampoules fertiles.

\section{Réhabilitation du genre Bakalovaella BucuR, 1993, et discussion sur sa position phylogénétique}

Compte tenu de sa proximité morphologique avec le genre Montiella (L. \& J. MORELLET, 1922), un autre genre de "Neomereae" (i.e., Dasycladeae), le genre Bakalovaella Bucur, 1993, n'était pas accepté par la plupart des auteurs. En effet, dans les 2 cas, les latérales sont constituées d'une primaire stérile portant une secondaire stérile et une ampoule fertile.

Chronologiquement, $L$. et $\mathrm{J}$. MoRelLet (1922) créent le genre Montiella et les espèces $M$. macropora (type du genre) et $M$. munieri. RADOIČIć (1980) propose le transfert de l'espèce Cylindroporella elitzae BAKALOVA, 1971, dans le genre Montiella, suivie de GRANIER (1990) qui propose le transfert de l'espèce Cylindroporella benizarensis FOURCADE et al. ex JAFFREZO in BASSOULLET et al., 1978, dans ce même genre. BuCUR (1993) crée le genre Bakalovaella fondé sur l'espèce Cylindroporella elitzae et y transfère également l'espèce Cylindroporella benizarensis. RADOIČIć (2006) crée l'espèce Montiella filipovici. D'un point de vue stratigraphique, les Bakalovaella ne sont connues qu'au Crétacé, alors que les Montiella ont été originellement décrites dans le Paléocène. Toutefois, cet argument stratigraphique ne saurait être retenu.

Les 2 espèces paléocènes de Montiella sont caractérisées par l'absence de minéralisation autour de l'axe principal et des longues primaires stériles qui $s^{\prime} y$ rattachent. Le second auteur (BuCUR, 1993, 2011 ; BUCUR et al., 2003, 2012) insiste sur cette absence de fossilisation des primaires stériles chez Montiella. Toutefois, c'est un caractère partagé avec de nombreux représentants d'une autre "Neomereae", i.e., la plupart des espèces fossiles du 
genre Neomeris J.V. Lamouroux, 1816, où cette minéralisation n'est préservée qu'exceptionnellement: par exemple, chez Neomeris (Neomeris) srivastavai GRANIER et al., 2013 (op. cit., Figs. 3b, 3.d, 4.a-b). À l'inverse, la minéralisation extracellulaire/intercellulaire (i.e., dans les cavités formées par les "replis" de la cellule) est plus poussée chez Bakalovaella ; aussi l'axe principal et les courtes primaires stériles qui s'y rattachent sont minéralisés. Ce n'est cependant pas ce caractère secondaire, i.e., le degré de minéralisation qui peut varier d'une espèce à l'autre, mais aussi entre individus d'une même espèce, qu'il faut retenir pour illustrer la différence fondamentale entre ces deux genres. L'organisation des latérales est similaire dans les deux genres. Toutefois, Bakalovaella est pourvue de primaires stériles courtes alors que Montiella est pourvue de primaires stériles relativement beaucoup plus longues. Conséquemment, le rapport d/D [= $\mathrm{d} /(\mathrm{d}+2 \mathrm{~L})]$ de Montiella devrait théoriquement être plus petit que celui de Bakalovaella. Toutefois, chez Montiella, ce n'est pas d (i.e., le diamètre de l'axe principal) qui peut être mesuré mais $\mathrm{d}^{*}$ (i.e., le diamètre de la cavité axiale). Dès lors, le rapport $d * / D$ de Montiella est plus grand que le rapport d/D de Bakalovaella. La longueur des latérales détermine aussi pour partie le volume intercellulaire susceptible d'être minéralisé. Ce volume est théoriquement bien plus important chez Montiella que chez Bakalovaella. Toutefois, chez Montiella, la minéralisation originelle (aragonitique) est limitée ; elle n'affecte que les ampoules fertiles et les secondaires stériles sous le cortex, mais pas l'axe principal, ni les primaires stériles.

Le genre Bakalovaella du Crétacé correspond à une forme intermédiaire entre le genre Barattoloporellopsis Granier et al., 2017, du Jurassique supérieur et le genre Montiella du Paléocène. Classé dans la famille des Dasycladaceae, il appartient à une lignée phylétique probablement issue de la famille des Diploporaceae. Pour ElLIOTT (1968), les formes choristospores (e.g., Cymopolia eochoristosporica ELLIOTT, 1968), i.e., les Dasycladaceae, dériveraient de formes cladospores, i.e., les Triploporellaceae, selon un modèle de "vases communicants" avec transfert de la fonction reproductrice d'une primaire fertile à une ampoule fertile qui va s'individualiser à l'extrémité distale de la dite primaire devenue stérile. L. EMBERGER (1968) propose un modèle d' "escalier mécanique" où l'ampoule fertile s'élève le long d'une primaire stérile ; ce qui peut être interprété aujourd'hui comme si les formes choristospores, i.e., les Dasycladaceae, étaient issues des formes goniospores, i.e., les Bornetellaceae. Notons que ces formes goniospores dériveraient elles-mêmes de formes cladospores (voir par exemple Zittelina massei Bucur et al., 2010), ce qui reviendrait à ajouter une bande de plus dans ce "coup de bil- lard" phylétique. Nous favorisons une troisième hypothèse, un temps envisagée mais aussitôt abandonnée par PIA (1920), qui considérait que les vestibules des Diploporaceae avaient pu donner naissance à des primaires stériles portant à leur extrémité distale une touffe de secondaires stériles et d'ampoules fertiles. Considérant la lignée phylogénétique probable Barattoloporellopsis $\longrightarrow$ Bakalovaella $\longrightarrow$ Montiella, c'est cette option que nous appelerons modèle du "piston élévateur" que nous favorisons. Dans cette hypothèse, les formes choristospores, i.e., les Dasycladaceae, dériveraient de formes ancestrales métaspondyles (à verticilles de latérales organisées en bouquets), i.e., les Diploporaceae. Accessoirement, les Polyphysaceae, dont de nombreuses formes présentent également un vestibule, pourraient également dériver des Diploporaceae.

Chez Bakalovaella, les verticilles successifs sont fortement imbriqués (BUCUR, 1993, 2011 ; Bucur et al., 2003 ; TAherpour Khalil AbAD et al., 2010 ), i.e., $\mathrm{e} / \mathrm{h} \approx 50 \%$, ce qui explique que ces formes aient été attribuées à Cylindroporella sensu Johnson, $1954^{[*]}$. Chez Montiella, ce rapport tend vers $100 \%$ (voir GÉNOT, 1987, PI. 6, figs. 5-6 ; PI. 31, figs. 1-12) ; de ce point de vue, Barattoloporellopsis du Jurassique supérieur est plus proche de Montiella du Paléocène que de $B a-$ kalovaella du Crétacé, ce qui explique que Barattoloporellopsis lusitanica, l'espèce-type du genre, ait été un temps attribuée à Heteroporella sensu PRATURLON, 1967.

\section{Révision du matériel aquitain}

Les sections d'algues illustrées par BouroulleC \& Deloffre (1968, Pl. 6, figs. 1-9) proviennent de deux niveaux stratigraphiques distincts du Crétacé inférieur :

1. un niveau basal (Bouroullec \& Deloffre, 1968, Pl. 6, figs. 1-2, 4-5, ?6, ?8 ; ici : Pl. 4, figs. A, E, G), d'âge Hauterivien probable (Granier \& ClaVel, 2019), où ces algues cylindroporelliformes sont associées à des Charophytes, et

2. un niveau un peu plus élevé (Bouroullec \& DelofFre, 1968, PI. 6, figs. 3, 7, 9), probablement d'âge Hauterivien, voire Barrémien inférieur (GRANIER \& CLAVEL, 2019), souvent en association avec Choffatella decipiens SCHLUMBERGER, Palaeodictyoconus cuvillieri (FourY), Deloffrella hauteriviana (MASSE), Falsolikanella danilovae RADOIČIĆ ex BARATTOLO et Pseudoactinoporella fragilis CONRAD.

Ces sections s'avèrent correspondre à deux, voire trois, espèces distinctes, dont une nouvelle pour les spécimens les plus anciens : cette espèce est décrite ici. Les spécimens les plus jeunes (Bouroullec \& Deloffre, 1968, Pl. 6, figs. 3, 7, 9) 
montrent des ampoules fertiles ouvertes à leur extrémité distale ; cette absence de minéralisation peut résulter de l'abrasion mécanique des spécimens ou, plus probablement, être liée au fait que les ampoules fertiles étaient au contact immédiat de la partie distale élargie des secondaires stériles formant le cortex. Le premier spécimen (Bouroullec \& Deloffre, 1968, PI. 6, fig. 3) avec son axe principal cylindrique et ses secondaires stériles larges est attribué à Bakalovaella elitzae (BAKALOVA, 1971); les deux autres spécimens (BouroulleC \& DelOFFre, 1968, PI. 6, figs. 7,9 ) avec des secondaires stériles fines (et un axe principal ondulé, non visible sur ces types de sections) sont attribués à Bakalovaella benizarensis (JAFFREZo in BASSOULLET et al., 1978).

L'une des principales caractéristiques de la nouvelle espèce (Bouroullec \& DelOFFRE, 1968, PI. 6 , figs. $1-2,4-6,8$ ) est que ses ampoules fertiles ne sont pas au contact de la partie distale élargie des secondaires stériles formant le cortex, ce qui a pour effet de ménager un espace intercellulaire qui va être le siège d'une minéralisation. Les ampoules fertiles paraissent plus enfoncées sous le cortex ; en réalité, ce caractère est dû à la différence de longueur entre l'ampoule fertile et la secondaire stérile issues d'une même primaire stérile.

\section{Systématique}

\section{Embranchement des Chlorophyta}

\section{Classe des Dasycladophyceae HOEK et al., 1995}

\section{Ordre des Dasycladales PAscher, 1931}

\section{Famille des Dasycladaceae} (KÜTZING, 1841)

\section{Tribu des Dasycladeae PIA, 1920 [ou Tribu des Neomereae (PIA, 1920)]}

\section{Genre Bakalovaella BucUR, 1993}

Générotype : Cylindroporella elitzae BAKALovA, 1971

Espèces incluses : Cylindroporella benizarensis FouRCADE et al. ex JAFFREZO in BASSOULLET et al., 1978 (PI. 5, figs. J-K ; PI. 6, figs. A-K) ; Bakalovaella deloffrei n.sp. ; Cylindroporella elitzae BAKALOVA, 1971 (voir par exemple TAHERPOUR KHALIL ABAD et al., 2010, Fig. 8 ; ici : Pl. 3, fig. H) ; Montiella filipovici RADOIČIć, 2006. Sous réserve de révisions, les espèces Cylindroporella faronensis MASSE et al., 1999, et C. massiliana MASSE et al., 1999, pourraient également se rattacher à ce genre.

\section{Bakalovaella deloffrei n.sp.}

(PI. 1, figs. A-L ; PI. 2, figs. A-N ; PI. 3, figs. $\mathrm{H}$; PI. 4, figs. A-J ; PI. 5, figs. A-I, L-M)

\section{Synonymie :}

1966.- Cylindroporella cf. sudgeni.- BOLTENHAGEN, PI. 2, fig. 10

1968.- Cylindroporella cf. sudgeni.- BOUROULLEC \& DELOFFRE, PI. 6, figs. 1 (ici : PI. 4, fig. A), 2, 4 (ici : PI. 4, fig. E), 5 (ici : Pl. 4, fig. G), 6, 8

1968.- Pianella dinarica.- Bouroullec \& Deloffre, PI. 7, fig. 3 (ici : Pl. 5, fig. F)

1970.- Cylindroporella cf. sudgeni.- BOUROULLEC \& DELOFFRE, PI. 2d, fig. 24

2019.- Bakalovaella deloffrei.- GRANIER \& CLAVEL, Figs. 5.U (ici : Pl. 1, fig. A), 5.V (ici : Pl. 2, fig. C)

Origine du nom : Cette nouvelle espèce est dédiée à la mémoire de Raoul Deloffre (19312018), géologue pétrolier et micropaléontologue, animateur avec son ami Alain PoIGNANT (19312011) du "Groupe Français d'Étude des Algues Fossiles".

Holotype et localité-type : Pl. 1, fig. A, section oblique de la partie fertile, lame mince $3669,5 \mathrm{~m}$, carotte 68, Lacq 104 (WGS84 43⒉ 13.4"N 0॰38'19.6"W).

Paratypes: Pl. 1, figs. G-H ; PI. 2, fig. C, autres sections obliques dans la même lame que I'holotype.

Diagnosis (English version): Small representative of the genus Bakalovaella Bucur, 1993. Subcylindrical main axis bearing deeply imbricated verticils of 5 to 8 laterals each. Laterals consisting of a short sterile primary followed by a fertile ampulla and a sterile secondary much longer than the ovoid ampulla. Abrupt distal widening of the sterile secondaries to form a cortex.

Diagnose: Petite forme attribuée au genre Bakalovaella Bucur, 1993. Axe principal subcylindrique portant des verticilles fortement imbriqués comportant chacun de 5 à 8 latérales. Latérales constituées par une courte primaire stérile suivie d'une ampoule fertile et d'une secondaire stérile bien plus longue que l'ampoule ovoïde. Élargissement distal abrupt des secondaires stériles pour former un cortex.

Description et dimensions : Il s'agit d'une petite forme avec $D=705 \pm 155 \mu \mathrm{m}$ ( 7 mesures), $d=140 \pm 60 \mu \mathrm{m}$ (9 mesures), $\mathrm{e}=290 \pm$ $70 \mu \mathrm{m}$ ( 7 calculs) et $d / D=18 \pm 9 \%$ ( 7 calculs). Le plus long spécimen mesuré dépasse $2 \mathrm{~mm}$. La plupart des sections observées correspondent à la partie fertile du thalle mais une partie pédonculaire stérile est parfois visible (PI. 1, figs. C-E ; PI. 2, figs. A-F ; Pl. 4, figs. A-B, $\mathrm{H}$; Pl. 5, figs. A, C, $\mathrm{E}, \mathrm{L})$. 
Les primaires stériles sont très courtes, souvent moins de $65 \mu \mathrm{m}\left(\mathrm{I}_{1}\right)$, pour une cinquantaine de $\mu \mathrm{m}$ de diamètre $\left(p_{1}\right)$. Les ampoules fertiles sont ovoïdes avec un diamètre pouvant atteindre $190 \mu \mathrm{m}\left(\mathrm{p}_{\mathrm{f}}\right)$ pour un allongement pouvant atteindre $250 \mu \mathrm{m}\left(\mathrm{I}_{\mathrm{f}}\right)$. Les secondaires stériles sont relativement longues, atteignant et dépassant souvent $350 \mu \mathrm{m}\left(\mathrm{I}_{2}\right)$, pour une cinquantaine de $\mu \mathrm{m}$ de diamètre $\left(p_{2}\right)$, à l'exception de leur partie distale abruptement élargie pour atteindre plus de $250 \mu \mathrm{m}$ de diamètre. L'espacement (e) des verticilles, de l'ordre de $150 \mu \mathrm{m}$, représente pratiquement la moitié de la hauteur (h) d'un verticille de l'ordre de $300 \mu \mathrm{m}$, i.e., e/h $\approx 50 \%$.

La minéralisation originelle en aragonite n'a pas été préservée. Les cristaux de sparite de certains spécimens, notamment ceux de Ousse 1 ( $\mathrm{PI}$. 4, figs. A-J ; PI. 5, figs. A-D, F-I, L), sont de teinte jaunâtre, indice d'un remplacement de l'aragonite par une mosaïque calcitique ; les cristaux de sparite des autres spécimens sont hyalins et correspondent à un ciment de calcite drusique remplissant la cavité de dissolution du manchon aragonitique de ces algues.

Dans quelques rares sections ( $\mathrm{PI} .1$, figs. $\mathrm{H}, \mathrm{J}-$ $\mathrm{K}$; PI. 3, figs. C-?D), on observe dans certaines ampoules fertiles, voire des latérales stériles, de petites structures subsphériques qui pourraient être interprétées comme des cystes. Cette observation (à confirmer) pourrait apporter plus de crédit au lien possible de la filiation entre Bakalovaella et Barattoloporellopsis.

\section{Remerciements}

Le premier auteur (B.G.) remercie ses nombreux anciens collègues de elf-Aquitaine et de TOTAL pour avoir facilité le prêt de matériel. Cette publication est dédiée à la mémoire de feu Raoul Deloffre décédé le 17 janvier 2018.

\section{Références bibliographiques}

BAKalova D. (1971).- Nouvelles espèces de Dasycladaceae (Algae) dans les sédiments urgoniens du Prébalkan central.- Bulletin of the Geological Institute, Bulgarian Academy of Sciences - Committee of Geology (Series Paleontology), Sofia, vol. XX, p. 123-127.

Bassoullet J.-P., Bernier P., Conrad M.A., DelofFRE R. \& JAFFREZO M. (1978).- Les algues Dasycladales du Jurassique et du Cretacé.- Géobios, Villeurbanne, Mémoire spécial 2, 330 p.

BoltenHAgen C. (1966).- Contribution à l'étude stratigraphique et structurale du flanc nord de l'anticlinal de Saint-Palais (Basses-Pyrénées).Thèse Docteur 3e Cycle, Université de Paris, 248 p.
Bouroullec J. \& Deloffre R. (1968).- Les algues du Néocomien d'Aquitaine.- Bulletin du Centre de Recherches de Pau - SNPA, vol. 2, no 2, p. 213-261.

Bouroullec J. \& Deloffre R. (1970).- Intrprétation sédimentologique et paléogéographique, par microfaciès, du Crétacé inférieur basal d'Aquitaine Sud-Ouest.- Bulletin du Centre de Recherches de Pau - SNPA, vol. 4, no 2, p. 381429.

B.R.G.M. (2018a).- Lacq 104. Fiche Synthétique AQI6400225. URL: http://ficheinfoterre.brgm. $\mathrm{fr} /$ InfoterreFiche/ficheBss.action?id=BSS002H PCY (dernier accès le 12/01/2019)

B.R.G.M. (2018b).- Ousse 1. Fiche Synthétique 10305X0009/OUS1. URL: http://ficheinfoterre. brgm.fr/InfoterreFiche/ficheBss.action?id=BSS 002KAWL (dernier accès le 12/01/2019)

BUCUR I.I. (1993).- Some new or poorly known calcareous algae (Dasycladales, Gymnocodiaceae) in the Lower Cretaceous deposits from the Resita-Moldava Noua Zone (Southern Carpathians, Romania).- Revista española de Micropaleontología, vol. XXV, p. 93-126.

BUCUR I.I. (2011).- Early Barremian dasycladalean algae from Serre de Bleyton (Drôme, SE France).- Annalen des Naturhistorischen $\mathrm{Mu}$ seums in Wien (Serie A), Wien, vol. 113, p. 619-653.

BUCUR I.I., GRANIER B. \& SĂSĂRAN E. (2010).- Zittelina massei n.sp., a new dasycladacean alga from the Lower Cretaceous strata of Pădurea Craiului (Apuseni Mountains, Romania).- Facies, Erlangen, vol. 56, no 3, p. 445-457.

BucUR I.I., RASHIDI K. \& Senowbari-Daryan B. (2012).- Early Cretaceous calcareous algae from central Iran (Taft Formation, south of Aliabad, near Yazd).- Facies, Erlangen, vol. 58 , no 4, p. 605-636.

Bucur I.I., Senowbari-Daryan B. \& Majidifard M.R. (2003).- Neocomian microfossil association from the Taft Area near Yazd (Central Iran).Facies, Erlangen, vol. 49, no 1, p. 217-222.

ELLIOTT G.F. (1957).- New calcareous algae from the Arabian Peninsula.- Micropaleontology, vol. 3, p. 227-230.

ElLiotT G.F. (1968).- Permian to Palaeocene calcareous algae (Dasycladaceae) of the Middle East.- Bulletin of the British Museum (Natural History) Geology, Suppl. 4, p. 3-111.

EMBerger L. (1968).- Les plantes fossiles dans leurs rapports avec les végétaux vivants : éléments de paléobotanique et de morphologie comparée ( ${ }^{\mathrm{e}}$ édition).- Masson, Paris, 759 p. 
Fourcade É., Jaffrezo M., Jerez Mir L. et RodriGUEZ ESTRELLA T. (1972).- El Jurásico terminal y el Cretácico inferior de la Sierra de la Muela (provincia de Murcia). Consideraciones sobre las biozonas con Foraminiferos del AlbenseAptiense del Sureste de España. In : $30^{\circ}$ Anniversario E.N. ADARO.- Revista española de Micropaleontología, no extr., p. 215-248.

GÉNOT P. (1987).- Les Chlorophycées calcaires du Paléogène d'Europe Nord-Occidentale (Bassin de Paris, Bretagne, Cotentin, Bassin de Mons). - Thèse Doctorat d'État, Nantes, 500 p.

GRANIER B. (1989).- Zergabriella, un nouveau genre d'Algue Dasycladale du Portlandien-Valanginien.- Revue de Micropaléontologie, Paris, vol . 32, no 2, p. 126-133.

GRANIER B. (1990).- Révision de Cylindroporella benizarensis FOURCADE et al. ex JAFFREZO in BASSOULLET et al. 1978.- Revista española de Micropaleontología, vol. XXII, no 2, p. 305311.

Granier B. (2002).- Algues Dasycladales, nouvelles ou peu connues, du Jurassique supérieur et du Crétacé inférieur du Moyen-Orient - New or little known Dasyclad algae from Upper Jurassic and Lower Cretaceous series of the Middle East. In: BUCUR I.I. \& FILIPESCU S. (eds.), Research advances in calcareous algae and microbial carbonates.- Proceedings of the 4th IFAA Regional Meeting (Cluj-Napoca, August 29 - September 5, 2001), Presa Universitara Clujeana, Cluj-Napoca, p. 103-113.

GRANIER B. (2013).- Heteroporella ? paucicalcarea CONRAD, 1970, an Urgonian Dasycladalean alga revisited.- Carnets Geol., Madrid, Letter CG2013/01 (CG2013_L01), p. 59-65.

GRANIER B. (2018).- Cylindroporella sugdeni ELLIOTT, 1957, an Early Cretaceous MiddleEastern Dasycladalean alga - a revision.- Journal of Micropaleontology, vol. 37, p. 181-190.

Granier B., AzerÊdo A. \& RAMAlHo R. (2017).Taxonomic revision of Cylindroporella ? lusitanica RAMALHO, 1970: In search for the origins of the Family Dasycladaceae.- Island Arc, vol. 26, no 3, e12176, 9 p.

Granier B. \& Clavel B. (2019).- Revised dating of the major earliest Cretaceous transgression in $S$ Aquitaine (SW France).- Cretaceous Research, in press.

Granier B., DiAS-BRITo D. \& BucuR I.I. (2013).- A new mid-Cretaceous Neomeris (dasycladacean alga) from the Potiguar Basin, Brazil.- Facies, Erlangen, vol. 59, no 1, p. 221-230.
Granier B., Masse J.-P. \& Berthou P.-Y. (1995).Heteroporella lepina PRATURLON, 1967, revisited (followed by taxonomic notes on the so-called "Heteroporella" species).- Proceedings of the International Symposium and Field-Meeting "Alpine Algae '93".- Beiträge zur Paläontologie, Vienna, no 19 (1994), p. 129-141.

Granier B., Radoičić R. \& Drobne K. (2013).- Revision of the Jesse Harlan JoHnson Collection. Part 1. Some fossil Dasycladales from Guatemala.- Carnets Geol., Madrid, Article CG2013/ 07 (CG2013_A07), p. 281-301.

Johnson J.H. (1954).- Cretaceous Dasycladacea from Gillespie county, Texas.- Journal of Paleontology, vol. 25, p. 787-790.

Masse J.-P., Bucur I.I., Virgone A. \& Dalmasso H. (1999).- Nouvelles espèces de Dasycladales du Crétacé inférieur de Provence (SE France).Revue de Micropaléontologie, Paris, vol. 42, $\mathrm{n}^{\circ}$ 3, p. 231-243 (2 Pls.).

Moreletet L. \& Moreletet J. (1922).- Nouvelle contribution à l'étude des Dasycladacées tertiaires.- Mémoires de la Société géologique de France, Paris, t. XXV, fasc. 2, no 58, 35 p.

PIA J. (1920).- Die Siphoneae verticillatae vom Karbon bis zur Kreide.- Abhandlungen der zoologisch-botanischen Gesellschaft in Wien, Band XI, Heft 2, 259 p.

Praturlon A. (1967).- Heteroporella lepina, new dasyclad species from upper Cenomanian lower Turonian of Central Apennines.- Bollettino della Società Paleontologica Italiana, Modena, vol. 5 (1966), no 2, p. 202-205 (Pls. 5152).

RADOIČIĆ R. (1980).- Contribution to the reconsideration of a group of cylindroporelliform dasyclads.- Bulletin de l'Académie Serbe des Sciences et des Arts, vol. LXXII, no 20, p. 109-115.

RADOIČIĆ R. (2006).- Trinocladus divnae and Montiella filipovici - a new species (Dasycladales, green algae) from the Upper Cretaceous of the Mountain Paštrik (Mirdita Zone).- Geološki anali Balkanskoga poluostrva, Beograd, vol. 67 , p. 65-87.

Taherpour Khalil Abad M., Conrad M.A., Aryaei A.A. \& Ashouri A.R. (2010).- Barremian-Aptian Dasycladalean algae, new and revisited from the Tirgan Formation in the Kopet Dagh, NE Iran.- Carnets Geol., Madrid, vol. 10, no A05 (CG2010_A05), 13 p. 

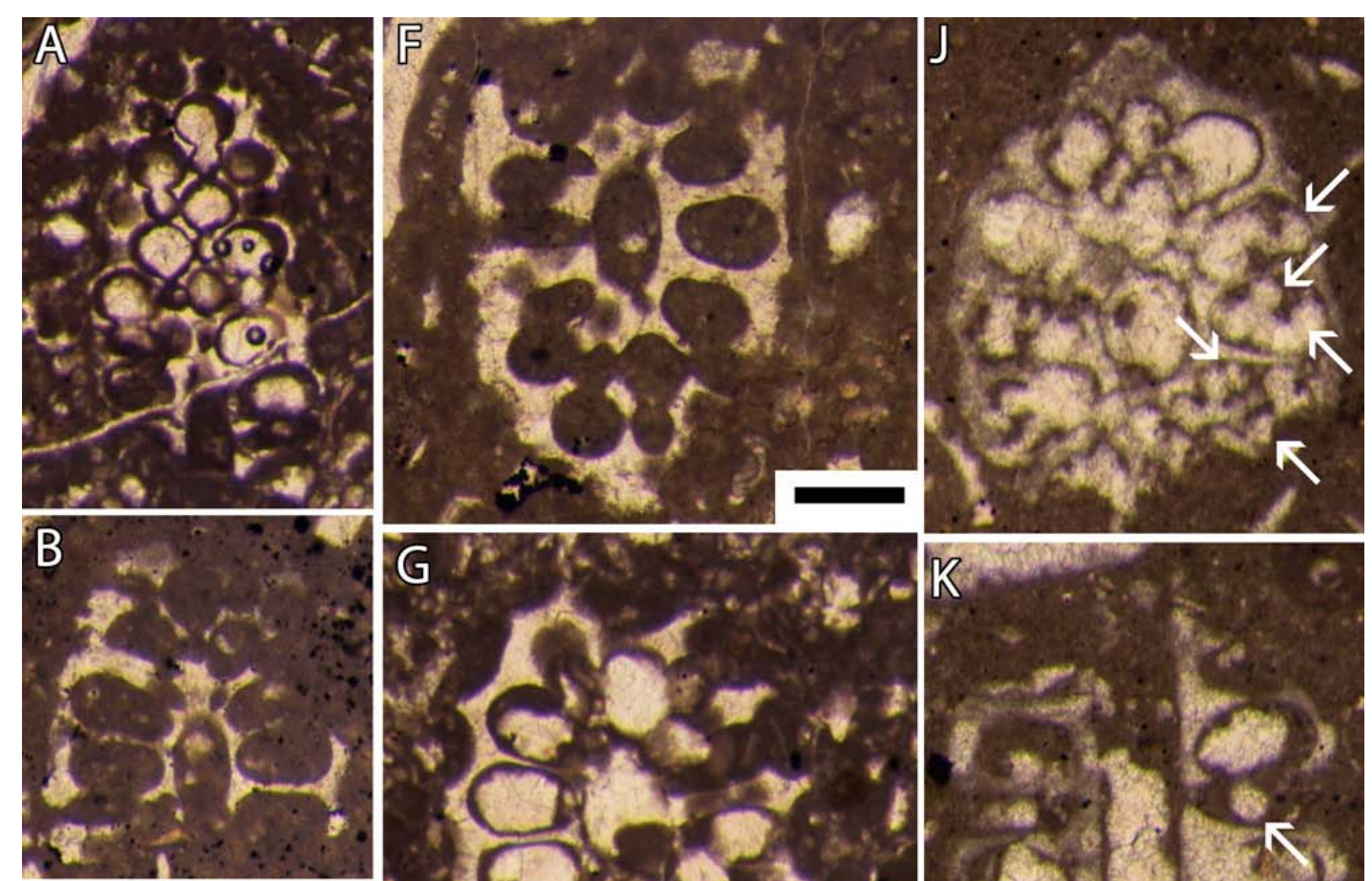

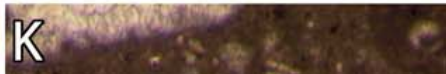
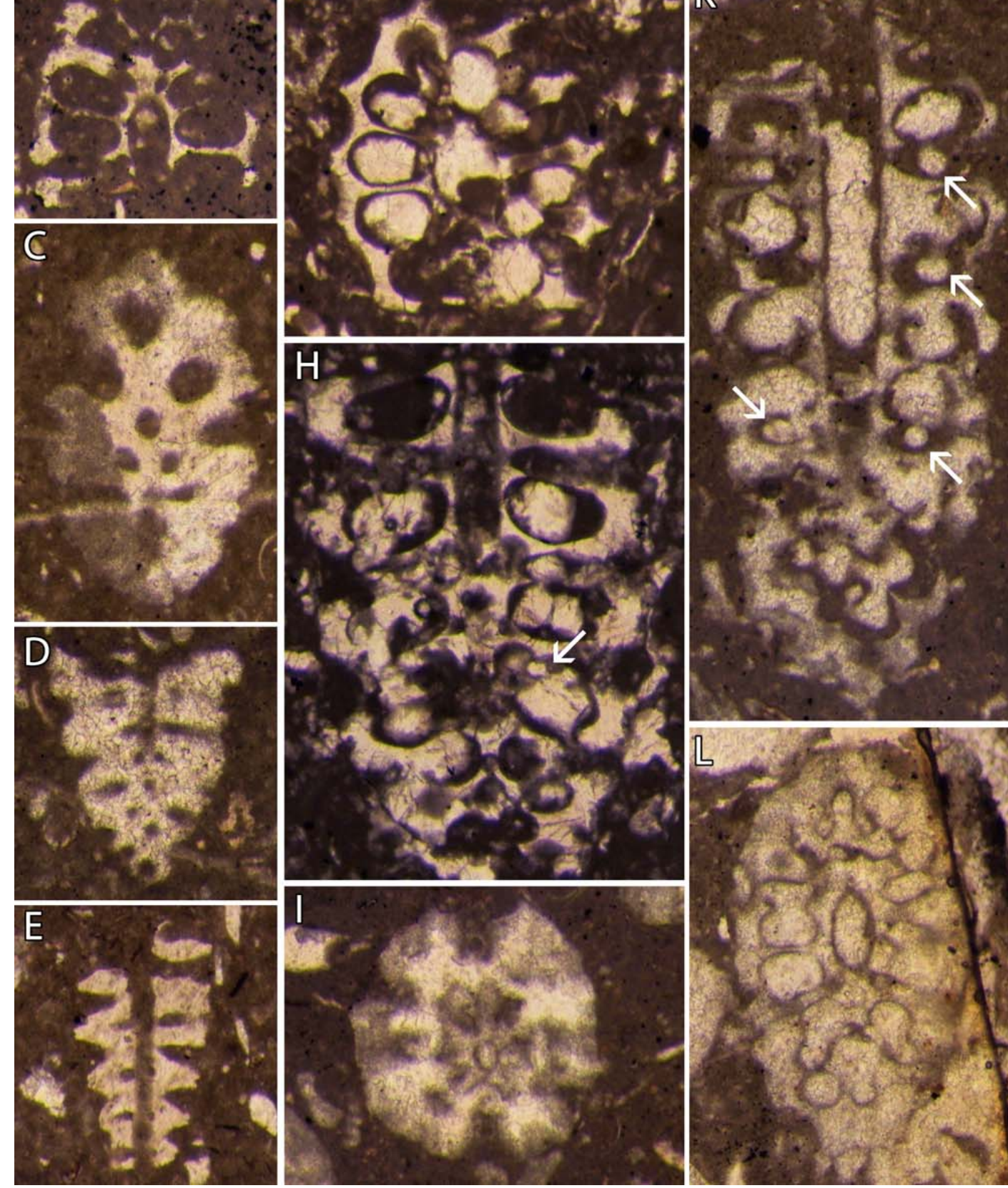

$\left.\cos ^{2}\right)^{3}$ is

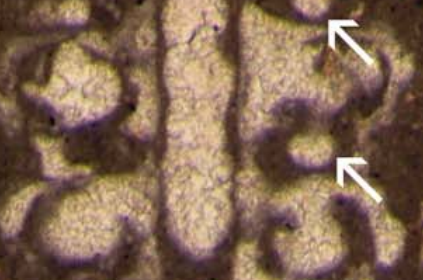

Nets: $(5)$

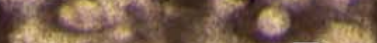
$x^{2}+2+5$

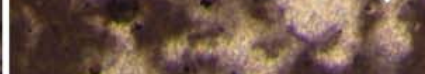
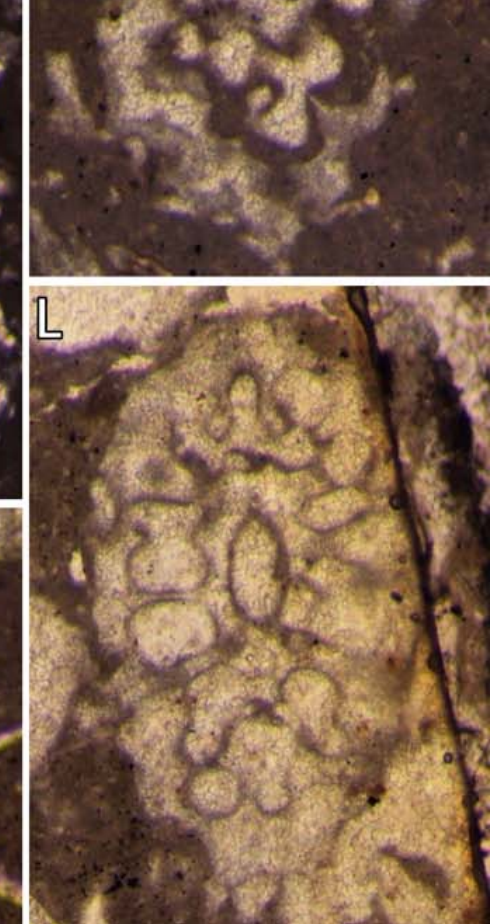


\section{Planches}

Planche 1 : Bakalovaella deloffrei n. sp.- A : holotype, section oblique de la partie fertile; B, F, J, L: sections obliques de la partie fertile ; $C$ : section oblique à la transition des parties stérile (basale) et fertile ; D : section oblique de la partie stérile basale; $\mathrm{E}$ : section axiale de la partie stérile basale; $\mathrm{F}$ : possible section subtransverse à la transition des parties stérile (basale) et fertile; G-H : paratypes, sections obliques de la partie fertile ; $\mathrm{K}$ : section subaxiale de la partie fertile. En $\mathrm{H}$, J et $\mathrm{K}$ (ainsi que sur la PI. 3, figs. C-?D), notez la présence de petites structures subsphériques (possibles ? cystes), identifiées par des flêches, dans certaines latérales et ampoules fertiles.

A, G-H : 3669,5 m, B-F : 3668,50 m, I-K : 3667,75 m (a), L : 3667,75 m (b), carotte 68, Lacq 104. Échelle graphique : $250 \mu \mathrm{m}$. 

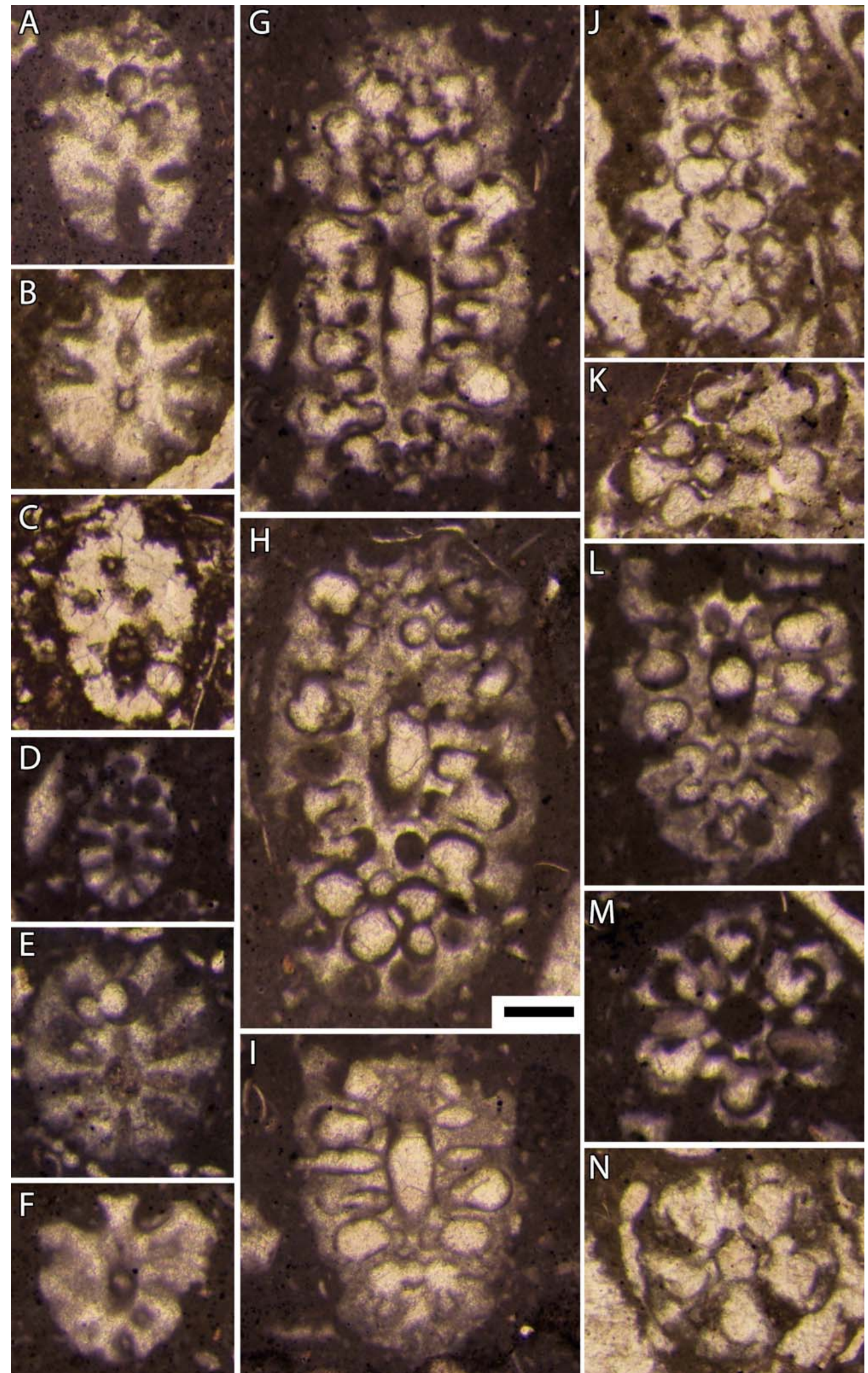
Planche 2 : Bakalovaella deloffrei n. sp.- A, D-F : sections obliques à la transition des parties stérile (basale) et fertile ; B : section oblique de la partie stérile basale ; C : paratype, section oblique de la partie stérile basale ; G-I, $\mathrm{L}$ : sections obliques de la partie fertile; J-K: sections tangentielles de la partie fertile; $\mathrm{M}-\mathrm{N}$ : sections subtransverses de la partie fertile. En J et $\mathrm{K}$, notez l'absence de calcification aux contacts entre latérales secondaires stériles et ampoules fertiles adjacentes (d'où la morphologie caractéristique en section des parties calcifiées).

A, D-I, L-M : 3667,75 m (a), B, J-K, N : 3667,75 m (b), C : 3669,5 m, carotte 68, Lacq 104. Échelle graphique : 250 $\mu \mathrm{m}$. 

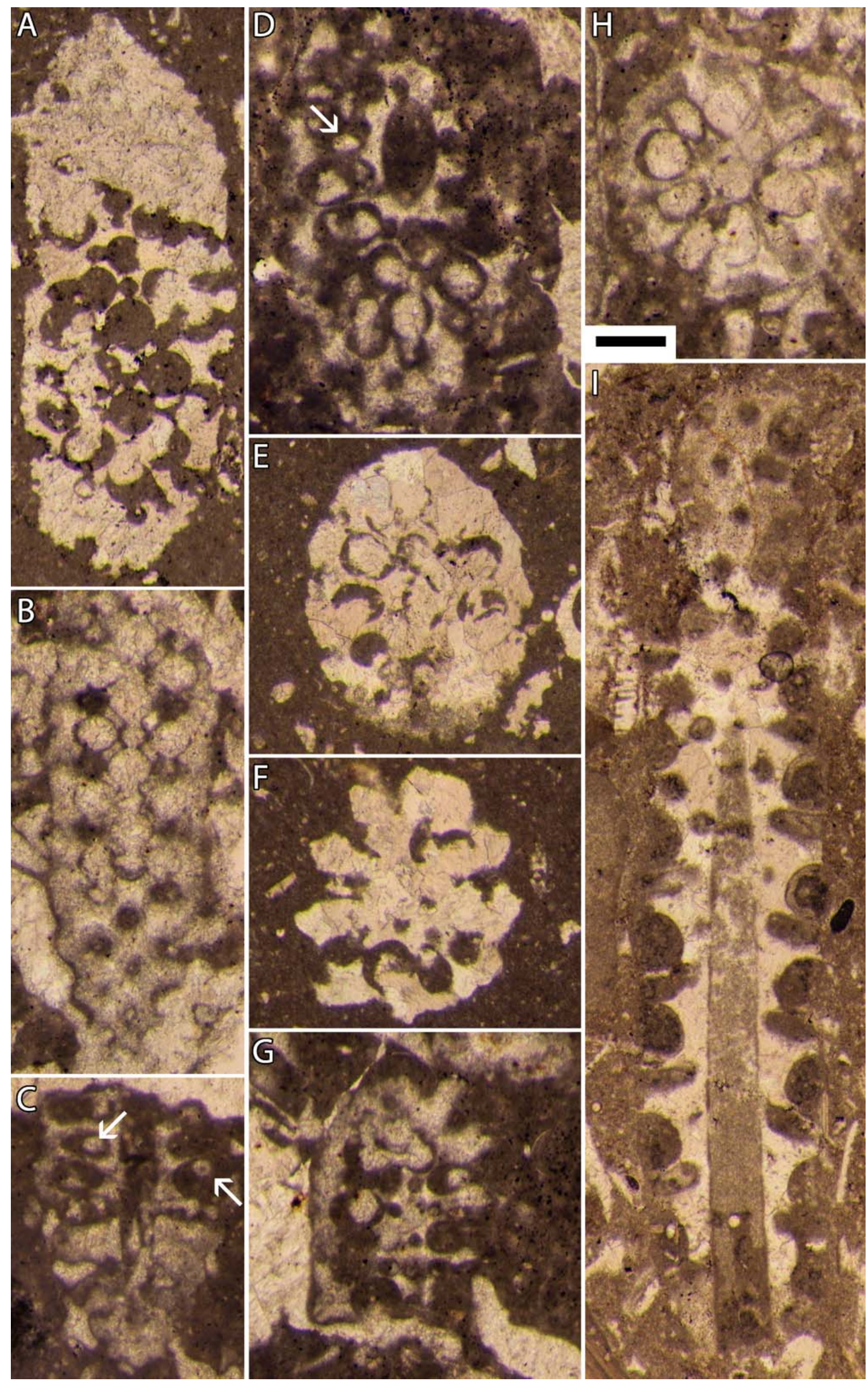
Planche 3 : Bakalovaella deloffrei n. sp.- A-B : sections tangentielles de la partie fertile ; C-E, G : sections obliques de la partie fertile ; $F$ : section subtransverse de la partie fertile ; $H$ : section subtransverse de la partie fertile. En $F$, notez l'élargissement relativement abrupt de la partie distale des latérales secondaires stériles pour former un cortex. Bakalovaella elitzae.- I : section subaxiale de la partie fertile.

A, E-F : intervalle "3646,5 m-3657,5 m", carotte 66, Lacq 104 ; B-D, G-H : 3667,75 m (b), carotte 68, Lacq 104 ; I : intervalle "3470,5 m-3476 m", carotte 40, Lacq 104. Échelle graphique : $250 \mu \mathrm{m}$. 

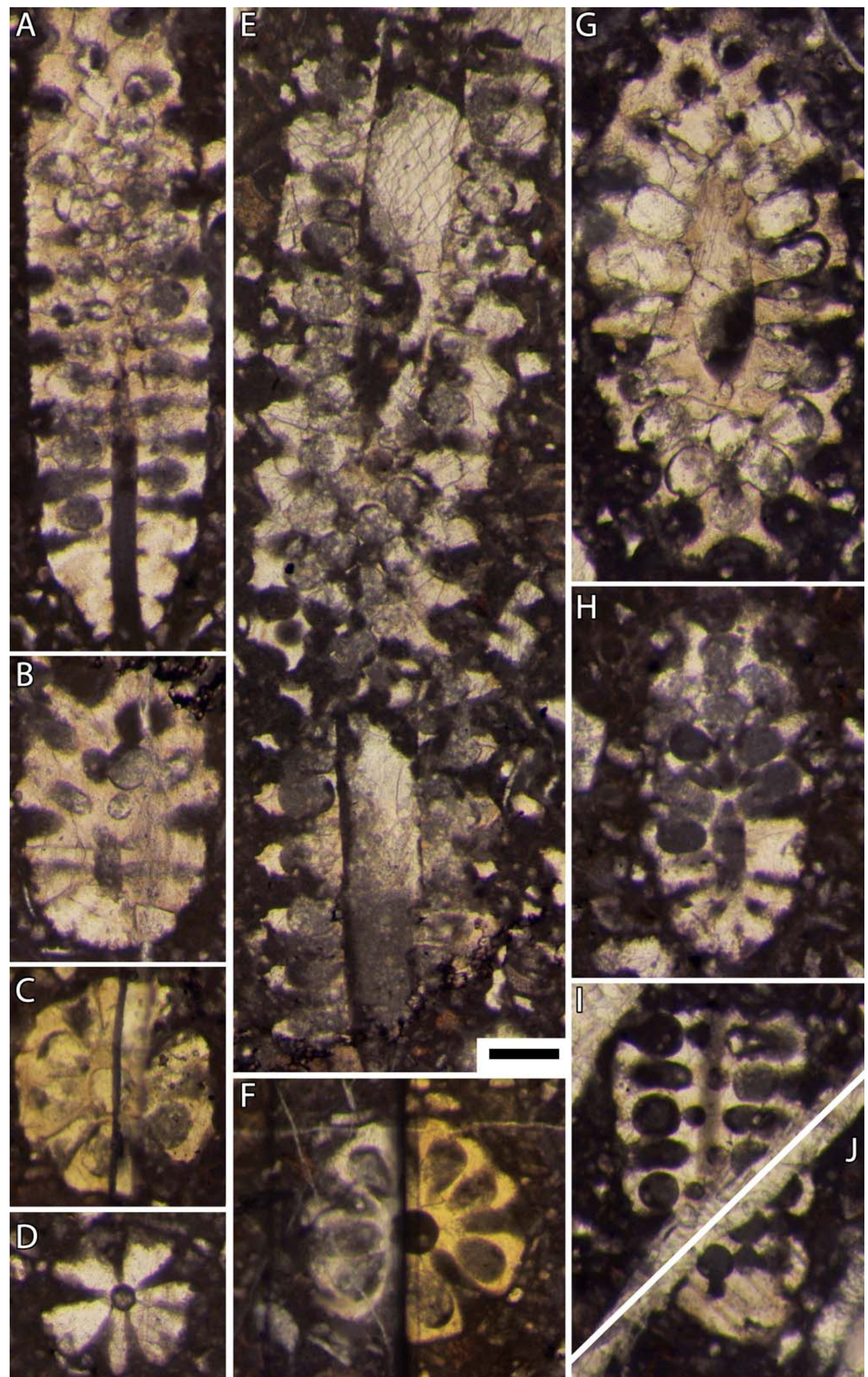
Planche 4 : Bakalovaella deloffrei n. sp.- A : section subaxiale avec la transition des parties stérile (basale) et fertile ; $\mathrm{B}, \mathrm{H}$ : sections obliques à la transition des parties stérile (basale) et fertile ; $\mathrm{C}, \mathrm{F}$ : sections subtransverses de la partie fertile; $D$ : section subtransverse de la partie stérile ; $E$ : section axiale de la partie fertile; $G$ : section oblique de la partie fertile; I-J : sections tangentielles profondes de la partie fertile. En E, notez l'élargissement relativement abrupt de la partie distale des latérales secondaires stériles pour former un cortex, ainsi que la terminaison bifide de la calcification intercellulaire entre deux latérales secondaires stériles au niveau du cortex, initialement interprétées comme des "mamelons" par Bouroullec et Deloffre (1968, p. 233, Fig. 3).

A = PI. 6, fig. 1 in Bouroullec et Deloffre, $1968 ; E=$ PI. 6, fig. 4 in BouroulleC et Deloffre, $1968 ; \mathrm{G}=\mathrm{PI} .6$, fig. 5 in Bouroullec et Deloffre, 1968. A-J : 5123,75 m, carotte 9, Ousse 1. Échelle graphique : $250 \mu \mathrm{m}$. 


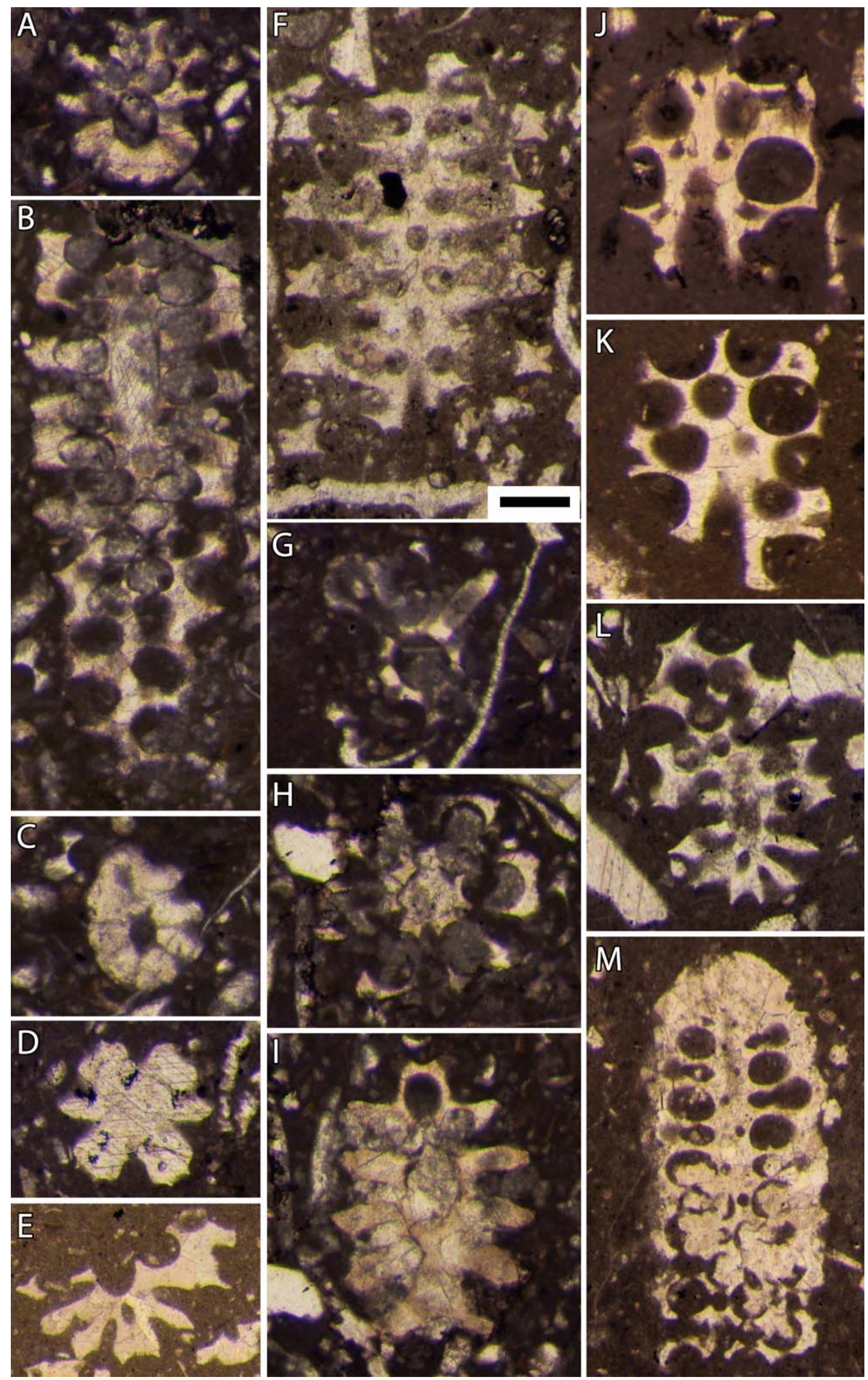


Planche 5 : Bakalovaella deloffrei n. sp.- A, C, E, L : sections obliques à la transition des parties stérile (basale) et fertile ; B, F, M : sections tangentielles de la partie fertile; $\mathrm{D}, \mathrm{G}-\mathrm{H}$ : sections subtransverses de la partie fertile ; I : section oblique de la partie fertile. Bakalovaella benizarensis.- J-K : sections obliques de la partie fertile.

A-C, G-I : 5123,75 m, carotte 9, Ousse 1 ; D, L : 5126,82 m, carotte 9, Ousse 1 ; E : intervalle "3646,5 m-3657,5 m", carotte 66, Lacq 104 ; F : 5129,35 m, carotte 9, Ousse 1 ; J : 3555,80 m, carotte 53, Lacq 104 ; K : intervalle "3546,50 m-3,554,80 m", carotte 52, Lacq $104 ;$ M : 3664 m, carotte 68, Lacq 104. Échelle graphique : $250 \mu \mathrm{m}$. 

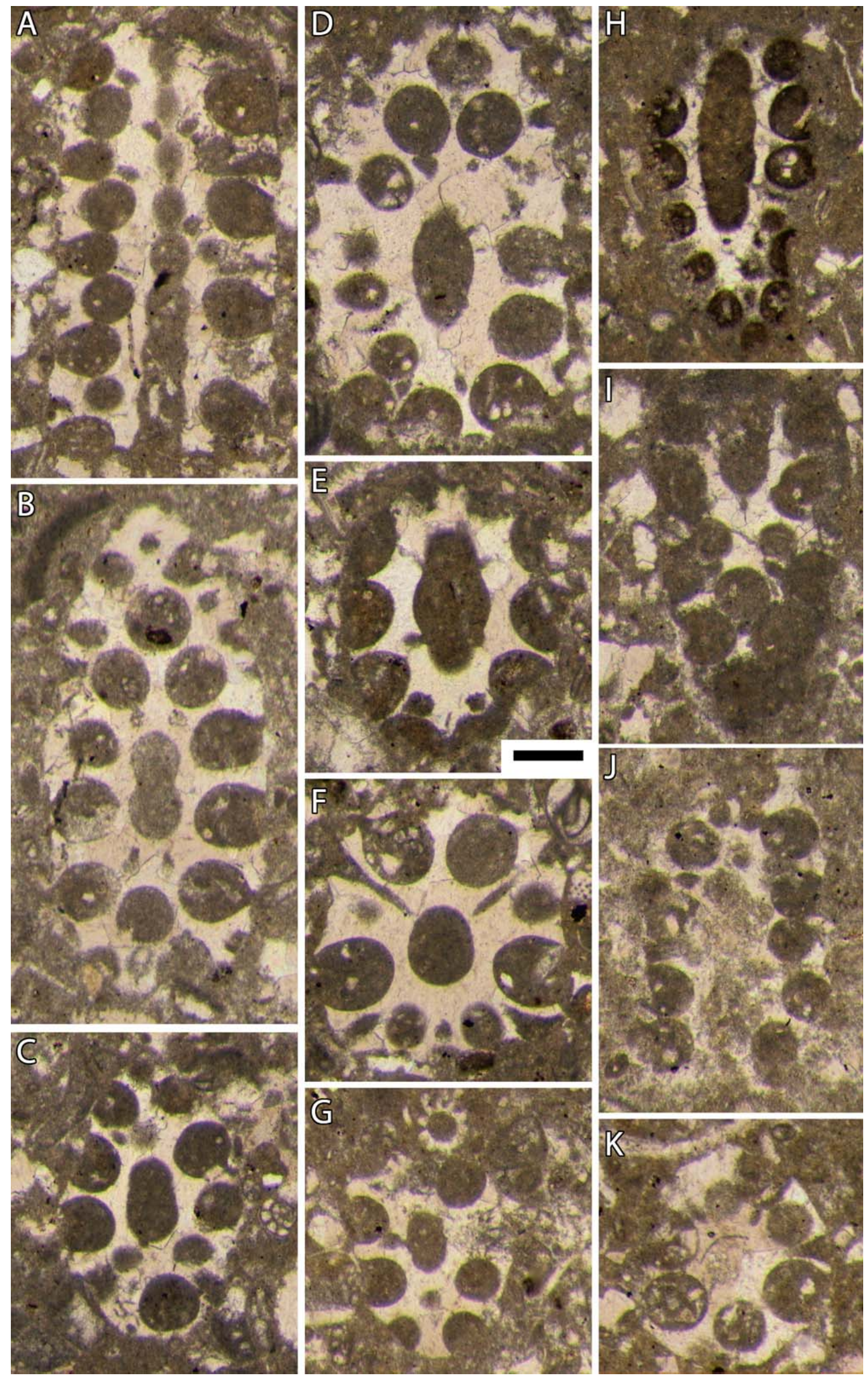
Planche 6 : Bakalovaella benizarensis.- A, H : sections subaxiales de la partie fertile ; B-E, G, I-J : sections obliques de la partie fertile ; $F, K$ : sections subtransverses de la partie fertile. En $K$, notez que w (i.e., le nombre de latérales par verticilles) $=6$.

A, lectotype = PI. 1, fig. 4 in Granier, $1990=$ PI. 3, fig. 5 in Fourcade et al., 1972 ; B = PI. 1, fig. 1 in Granier, 1990, et détail sur la PI. 1, fig. 9 in Granier, 1990 ; D = détail sur la PI. 1, fig. 7 in Granier, 1990 ; F = PI. 3 , fig. 4 in FourCADe et al., $1972 ; \mathrm{H}=$ détail sur la PI. 1, fig. 8 in GRANIER, 1990. A-K : lame mince G782, Collection FouRCADE, Benizar, Murcie (Espagne). Échelle graphique : $250 \mu \mathrm{m}$. 